\title{
TITLE:
}

\section{High energy neutrino flashes from far-ultraviolet and $X$-ray flares in gamma-ray bursts}

$\operatorname{AUTHOR}(\mathrm{S})$ :

Murase, K; Nagataki, S

\section{CITATION:}

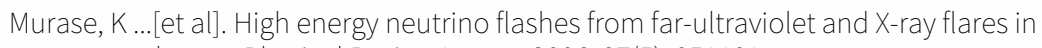
gamma-ray bursts. Physical Review Letters 2006, 97(5): 051101.

ISSUE DATE:

2006-08-04

URL:

http://hdl.handle.net/2433/50481

RIGHT:

Copyright 2006 American Physical Society 


\title{
High Energy Neutrino Flashes from Far-Ultraviolet and X-Ray Flares in Gamma-Ray Bursts
}

\author{
Kohta Murase $^{1}$ and Shigehiro Nagataki ${ }^{1,2}$ \\ ${ }^{1}$ Yukawa Institute for Theoretical Physics, Kyoto University, Oiwake-cho, Kitashirakawa, Sakyo-ku, Kyoto, 606-8502, Japan \\ ${ }^{2}$ KIPAC, Stanford University, P.O. Box 20450, MS 29, Stanford, California 94309, USA
}

(Received 21 April 2006; revised manuscript received 9 June 2006; published 2 August 2006)

\begin{abstract}
The recent observations of bright optical and $\mathrm{x}$-ray flares by the Swift satellite suggest these are produced by the late activities of the central engine. We study the neutrino emission from far-ultraviolet and x-ray flares under the late internal shock model. We show that the efficiency of pion production in the highest energy is comparable to or higher than the unity, and the contribution from such neutrino flashes to a diffuse very high energy neutrino background can be larger than that of prompt bursts if the total baryonic energy input into flares is comparable to the radiated energy of prompt bursts. These signals may be detected by IceCube and are very important because they have possibilities to probe the nature of flares (the baryon loading, the photon field, the magnetic field and so on).
\end{abstract}

DOI: 10.1103/PhysRevLett.97.051101

High energy neutrino emission from gamma-ray bursts (GRBs) has been expected in the context of the standard internal-external scenario of GRBs. Especially, since the prediction of Waxman and Bahcall [1], neutrino bursts in the internal shock model have been studied by several authors and neutrino afterglows in the external shock model have also been discussed [2,3].

The standard model of GRBs has succeeded in explaining many observations, but there were a few outstanding questions in the study of GRBs before the launch of the Swift satellite [see reviews, e.g., [4]]. The Swift satellite, which is an ideal mission to answer these questions, has presented indeed very fruitful results during the first several months of its operation. Especially in the x-ray band, Swift $\mathrm{x}$-ray telescope detected an x-ray afterglow for essentially every burst, which showed the surprising behaviors that are not straightforwardly expected in the pre-Swift era [5]. The early afterglow light curve has several surprising features such as an early steep decay, a follow-up shallower-than-normal decay, one or more x-ray flares and so on [see, e.g., [5]]. Especially, observations show that many bursts have large x-ray flares superimposed on the underlying afterglow. In the early afterglows of XRF 050406 and GRB 050502b, x-ray telescope detected mysterious strong x-ray flares, and some flares such as GRB 050607 and GRB 050904 have multiple flares [6]. These observational results suggest the existence of additional emission in the early afterglow phase besides the conventional forward shock emission.

In this Letter we calculate high energy neutrino emission from far-ultraviolet (FUV) and x-ray flares under the late internal shock model [7]. Our method of calculation using GEANT4 [8] is the same as in Murase and Nagataki [2], but quantitatively improved [9]. Now, large neutrino detectors such as IceCube, ANTARES, and NESTOR are being constructed [10]. In the near future, these detectors may detect high energy neutrino signals correlated with flares.

The model. - The flares typically happen hundreds of seconds after the trigger of prompt emissions or earlier. In
PACS numbers: 98.70.Rz, 95.85.Ry

some cases, they occur around a day after the burst. The observed typical time scale is larger than that of the prompt emission, which is $\delta t \sim\left(10-10^{3}\right)$ s [5,6]. The amplitudes of the flares are usually larger than the underlying afterglow component by a factor of several, but can be much larger. These can be even comparable to or higher than the prompt burst component which is typically $L_{\mathrm{X}}^{\mathrm{GRB}} \sim$ $\left(10^{49}-10^{52}\right) \mathrm{ergs} / \mathrm{s}$ around $(1-10) \mathrm{keV}$ band. Hereafter we take $L_{\max }=\left(10^{47}-10^{50}\right) \mathrm{ergs} / \mathrm{s}$ as a peak luminosity of far-ultraviolet and x-ray flares. Although some flares allow for the possibility of external shock processes, the general features of the flares suggest that this phenomenon is best interpreted as a late internal central engine activity [5]. The variability of some GRB afterglows implies that the engine may last much longer than the duration of the bursts [11], although a possible mechanism for reactivity of the central engine is unknown [12]. We suppose the late internal shock occurs a few minutes after the prompt $\gamma$-ray emission, powering a new unsteady relativistic outflow. Falcone et al. show that the case of GRB 050502b implies the late outflow has the smaller Lorentz factor, $\Gamma \lesssim 20$ [6]. Hence, we assume that the typical Lorentz factors of the ejected material are smaller than the prompt emission, setting $\Gamma_{s} \sim 10$ and $\Gamma_{f} \sim 100$ as the typical Lorentz factors of the slow and fast shells, respectively. We can estimate the Lorentz factor of the merged shell $\Gamma \approx \sqrt{\Gamma_{f} \Gamma_{s}} \simeq$ 30, and the Lorentz factor of the internal shocks can be estimated by $\Gamma_{\mathrm{sh}} \approx\left(\sqrt{\Gamma_{f} / \Gamma_{s}}+\sqrt{\Gamma_{s} / \Gamma_{f}}\right) / 2 \sim$ a few. This regenerated internal shocks are called as the late internal shocks [7]. The typical collision radius is expressed by commonly used relation, $r \approx 10^{14.5}(\Gamma / 30)^{2}[\delta t / 6(1+$ $z) \mathrm{s}] \mathrm{cm}$. Of course, it should be smaller than the deceleration radius. Actually the exact radiation mechanism producing the flares is unclear. Here, however, let us assume that flares are produced by the synchrotron emission to see typical parameters. The minimal Lorentz factor of electrons is estimated by $\gamma_{e, m} \approx \epsilon_{e}\left(m_{p} / m_{e}\right)\left(\Gamma_{\mathrm{sh}}-1\right)$. Since 
we can estimate the intensity of magnetic field by $B=$ $7.7 \times 10^{3} \mathrm{G} \epsilon_{B,-1}^{1 / 2}\left[\Gamma_{\mathrm{sh}}\left(\Gamma_{\mathrm{sh}}-1\right) / 2\right]^{1 / 2} L_{\mathrm{M}, 50}^{1 / 2} \Gamma_{30}^{-1} r_{14.5}^{-1}$, the observed break energy is $E^{b}=\hbar \gamma_{m}^{2} \Gamma e B / m_{e} c \sim 0.1 \mathrm{keV} \times$ $\epsilon_{e,-1}^{2} \epsilon_{B,-1}^{1 / 2}\left(\Gamma_{\mathrm{sh}}-1\right)^{5 / 2}\left(\Gamma_{\mathrm{sh}} / 2\right)^{1 / 2} L_{\mathrm{M}, 50}^{1 / 2} r_{14.5}^{-1} \quad$ [7]. Here, $L_{\mathrm{M}}$ is the outflow luminosity. Therefore, the typical emitted energy is in the soft x-ray band.

For numerical calculations, we simply set the break energy and adopt the power-law spectrum similarly to that of the case of the prompt emission. Although most of flares are actually well fitted by a Band function or cutoff power-law model [6], such a treatment does not change our results so much. We use the following expression in the comoving frame, $d n / d \varepsilon=n_{b}\left(\varepsilon / \varepsilon^{b}\right)^{-\alpha}$ for $\varepsilon^{\min }<\varepsilon<\varepsilon^{b}$ or $d n / d \varepsilon=n_{b}\left(\varepsilon / \varepsilon^{b}\right)^{-\beta}$ for $\varepsilon^{b}<\varepsilon<$ $\varepsilon^{\max }$, where we set $\varepsilon^{\mathrm{min}}=0.1 \mathrm{eV}$ because the synchrotron self-absorption will be crucial below this energy and $\varepsilon^{\max }=1 \mathrm{MeV}$ because the pair absorption will be crucial above this energy [13]. Actually we do not know the peak energy and the lower spectral index of many flares [7]. So we allow for the existence of far-ultraviolet-ray (FUV-ray) flares and take $\varepsilon^{b}=(0.01-0.1) \mathrm{keV}$ in the comoving frame. We assume $\alpha=1$ and set $\beta=2.2$ [5] similarly to the prompt emission. The photon energy density is $U_{\gamma}=\int d \varepsilon \varepsilon d n / d \varepsilon$. The magnetic energy density and the nonthermal proton energy density are expressed by $U_{B}=$ $\xi_{B} U_{\gamma}$ and $U_{p}=\xi_{\text {acc }} U_{\gamma}$, respectively. The nonthermal baryon loading factor $\xi_{\text {acc }}$ can be expressed by $\xi_{\text {acc }} \approx$ $\zeta_{p} 10\left(0.1 / \epsilon_{e}\right)$, where $\zeta_{p}$ is the proton acceleration efficiency. Roughly speaking, $\xi_{\text {acc }}=10$ and $\xi_{B}=1$ correspond to $\epsilon_{B}=0.1$ if $\zeta_{p} \sim 1$. We take $r \sim 10^{14.5-16} \mathrm{~cm}$ with $\Gamma \sim(10-50)$. Although we have done wide parameter surveys, we will hereafter set the width of the shell to $r / 2 \Gamma^{2}=4.5 \times 10^{11-12} \mathrm{~cm}$ according to $\delta t=(30-300) \mathrm{s}$ at $z=1$, and show the two cases of $r=10^{14.9} \mathrm{~cm}$ with $\Gamma=30$ and $r=10^{15.3} \mathrm{~cm}$ with $\Gamma=15$.

To obtain the pion production rate and estimate the maximal energy of accelerated protons, we need to take into account following various cooling time scales. We consider the synchrotron cooling time written by $t_{\text {syn }}=$ $3 m_{p}^{4} c^{3} / 4 \sigma_{\mathrm{T}} m_{e}^{2} \varepsilon_{p} U_{B}$, the inverse-Compton (IC) cooling time which is given by Jones [14], the adiabatic cooling time comparable to the dynamical time, and the photomeson cooling time which is evaluated by

$$
t_{p \gamma}^{-1}\left(\varepsilon_{p}\right)=\frac{c}{2 \gamma_{p}^{2}} \int_{\bar{\varepsilon}_{\mathrm{th}}}^{\infty} d \bar{\varepsilon} \sigma_{p \gamma}(\bar{\varepsilon}) \kappa_{p}(\bar{\varepsilon}) \bar{\varepsilon} \int_{\bar{\varepsilon} / 2 \gamma_{p}}^{\infty} d \varepsilon \varepsilon^{-2} \frac{d n}{d \bar{\varepsilon}},
$$

where $\bar{\varepsilon}$ is the photon energy in the rest frame of proton, $\gamma_{p}$ is the proton's Lorentz factor, $\kappa_{p}$ is the inelasticity of proton, and $\bar{\varepsilon}_{\text {th }} \approx 145 \mathrm{MeV}$ is the threshold photon energy for photomeson production. From various time scales, we can estimate the total cooling time scale by $t_{p}^{-1} \equiv t_{p \gamma}^{-1}+$ $t_{\mathrm{syn}}^{-1}+t_{\mathrm{IC}}^{-1}+t_{\mathrm{ad}}^{-1}$. We believe that not only electrons but also protons can be accelerated by the first-order Fermi acceleration mechanism and assume $d n_{p} / d \varepsilon_{p} \propto \varepsilon_{p}^{-2}$. By the condition $t_{\text {acc }}<t_{p}$, we can estimate the maximal energy of accelerated protons. Here, we take the acceleration time scale by $t_{\text {acc }} \sim \varepsilon_{p} / e B c$. We also set the minimal energy of protons to $10 \mathrm{GeV}$ because this would be around $\sim \Gamma_{\mathrm{sh}} m_{p} c^{2}$. One of numerical results is shown in Fig. 1.

Let us evaluate $f_{p \gamma} \equiv t_{\text {dyn }} / t_{p \gamma}$ by $\Delta$-resonance approximation. After performing the second integral in Eq. (1), we can approximate by

$$
t_{p \gamma}^{-1} \simeq \frac{U_{\gamma}}{2 \varepsilon^{b}} c \sigma_{\Delta} \kappa_{p}\left(\bar{\varepsilon}_{\Delta}\right) \frac{\Delta \bar{\varepsilon}}{\bar{\varepsilon}_{\Delta}}\left\{\begin{array}{l}
\left(\bar{\varepsilon}_{\Delta} / 2 \gamma_{p} \varepsilon^{b}\right)^{-(\beta-1)} \\
\left(\bar{\varepsilon}_{\Delta} / 2 \gamma_{p} \varepsilon^{b}\right)^{-(\alpha-1)}
\end{array},\right.
$$

where $\sigma_{\Delta} \sim 5 \times 10^{-28} \mathrm{~cm}^{2}, \quad \kappa_{p}\left(\bar{\varepsilon}_{\Delta}\right) \sim 0.2$, and $\bar{\varepsilon}_{\Delta} \sim$ $0.3 \mathrm{GeV}$, and $\Delta \bar{\varepsilon} \sim 0.2 \mathrm{GeV}$ [1]. Here, we have included the effect of multipion production and high inelasticity which is moderately important, and multiplied by a factor of $\sim(2-3)$ in Eq. (2) [2]. Hence, we can obtain

$$
f_{p \gamma} \simeq 10 \frac{L_{\max , 49}}{r_{14.5} \Gamma_{30}^{2} E_{\mathrm{keV}}^{b}} \begin{cases}\left(E_{p} / E_{p}^{b}\right)^{\beta-1} & \left(E_{p}<E_{p}^{b}\right) \\ \left(E_{p} / E_{p}^{b}\right)^{\alpha-1} & \left(E_{p}^{b}<E_{p}\right)\end{cases}
$$

where $E_{p}^{b} \simeq 0.5 \bar{\varepsilon}_{\Delta} m_{p} c^{2} \Gamma^{2} / E^{b}$ is the proton break energy. From Eq. (3), we can conclude that a significant fraction of high energy accelerated protons cannot escape from the source without photomeson productions. In the case of bright $\mathrm{x}$-ray flares whose luminosity is larger than $10^{49} \mathrm{ergs} / \mathrm{s}$ and FUV-ray flares, almost all protons accelerated to the very high energy region will be depleted.

Neutrino spectrum and flux.-As in the case of the prompt emission, we can expect high energy neutrino flashes from one GRB event only if the flare is nearby or energetic. In Fig. 2, we show an example of the observed neutrino flux from the source at $z=0.1$. The expected muon events for above $\mathrm{TeV}$ energy neutrinos are $N_{\mu}=$ 0.02 events in the case of $\mathrm{x}$-ray flare (A) in Fig. 2. We can expect $N_{\mu}=1.4$ events when the flare is energetic and more nonthermally baryonic [FUV-ray flare (B)]. Note that, in the case of an energetic flare, the maximal neutrino energy will become small because the highest energy protons suffer from the photomeson cooling very much.

We can estimate a diffuse neutrino background from FUV/x-ray flares for specific parameter sets under the

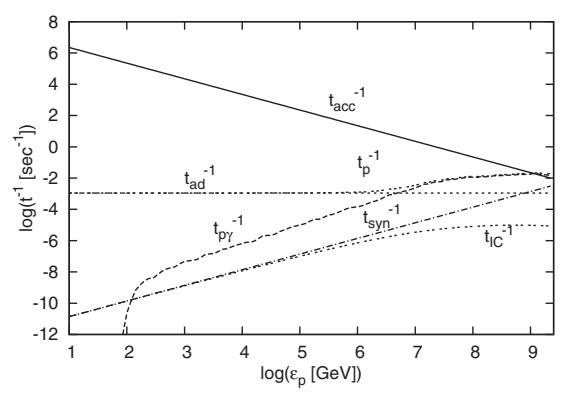

FIG. 1. Various cooling time scales and the acceleration time scale for FUV-ray flares with $L_{\max }=10^{49} \mathrm{ergs} / \mathrm{s}, \xi_{B}=1$, and $r=10^{14.9} \mathrm{~cm}$ with $\Gamma=30$. Note that energy scale is measured in the shell comoving frame. 


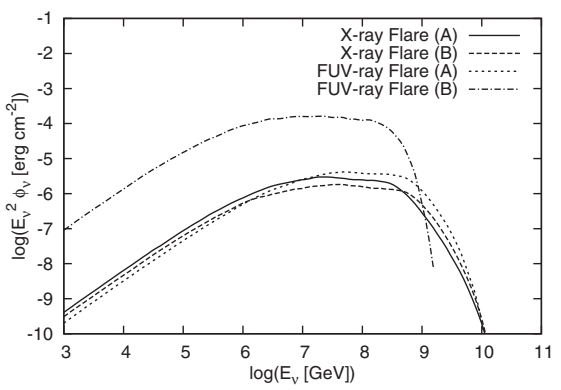

FIG. 2. The observed muon-neutrino $\left(\nu_{\mu}+\bar{\nu}_{\mu}\right)$ spectra for one GRB event at $z=0.1$. X-ray flare (A): $L_{\max }=$ $10^{49} \mathrm{ergs} / \mathrm{s}, \xi_{B}=1, \xi_{\text {acc }}=10$, and $r=10^{14.9} \mathrm{~cm}$ with $\Gamma=$ 30. X-ray flare (B): $L_{\text {max }}=10^{48} \mathrm{ergs} / \mathrm{s}, \xi_{B}=1, \xi_{\text {acc }}=10$, and $r=10^{15.3} \mathrm{~cm}$ with $\Gamma=15$. FUV-ray flare (A): $L_{\max }=$ $10^{48} \mathrm{ergs} / \mathrm{s}, \xi_{B}=1, \xi_{\mathrm{acc}}=10$, and $r=10^{15.3} \mathrm{~cm}$ with $\Gamma=$ 15. FUV-ray flare (B): $L_{\max }=10^{50} \mathrm{ergs} / \mathrm{s}, \xi_{B}=0.1, \xi_{\text {acc }}=$ 30 , and $r=10^{14.9} \mathrm{~cm}$ with $\Gamma=30$.

standard $\Lambda$ CDM cosmology $\left(\Omega_{\mathrm{m}}=0.3, \Omega_{\Lambda}=0.7 ; H_{0}=\right.$ $71 \mathrm{~km} \mathrm{~s}^{-1} \mathrm{Mpc}^{-1}$ ). Assuming that the long GRB rate traces the star formation rate (SFR), we shall use the SF3 model of Porciani and Madau combined with the normalization of overall GRB rates obtained by Guetta et al. [15]. The choice of $z_{\max }$ which should be larger than 6.3 does not affect our results so much, so we take $z_{\max }=11$, which is the epoch when the reionization is likely to occur. X-ray flares are detected in at least (1/3-1/2) of Swift GRBs [5]. So we can expect that flares are common in GRBs. Here, introducing the ratio of the energy emitted by flares to that of the prompt emission, $f_{\mathrm{F}} \equiv E_{\mathrm{flare}} / E_{\mathrm{GRB}}$, let us estimate the neutrino flux analytically. First, we can express the total number spectrum of accelerated protons, using $\varepsilon_{p \text {,max }} \sim$ $10^{9} \mathrm{GeV}$,

$$
E_{p}^{2} \frac{d N_{p}}{d E_{p}} \simeq 1.6 \times 10^{50} N f_{\mathrm{b}} \xi_{\mathrm{acc}, 10} L_{\max , 49}\left(\frac{r_{14.5}}{\Gamma_{30}^{2}}\right) \mathrm{erg},
$$

where $f_{b}$ is the beaming factor and $N$ is the number of flares. Since we have already estimated $f_{p \gamma}$ in Eq. (3), by replacing $2.5 \mathrm{~N} f_{b} L_{\max } r / \Gamma^{2} c$ with the total emitted energy from flares $E_{\text {flare }}$, we can estimate the diffuse neutrino background flux from flares as follows,

$$
\begin{aligned}
E_{\nu}^{2} \Phi_{\nu} \sim & \frac{c}{4 \pi H_{0}} \frac{1}{4} \min \left[1, f_{p \gamma}\right] E_{p}^{2} \frac{d N_{p}}{d E_{p}} R_{\mathrm{GRB}}(0) f_{z} \\
\simeq & 6 \times 10^{-10} \mathrm{GeV} \mathrm{cm}{ }^{-2} \mathrm{~s}^{-1} \mathrm{str}^{-1} \min \left[1, f_{p \gamma}\right] \\
& \times f_{\mathrm{F}} \xi_{\mathrm{acc}} E_{\mathrm{GRB}, 51}\left(\frac{R_{\mathrm{GRB}}(0)}{20 \mathrm{Gpc}^{-3} \mathrm{yr}^{-1}}\right)\left(\frac{f_{z}}{3}\right),
\end{aligned}
$$

where $f_{z}$ is the correction factor for the possible contribution from high redshift sources and $R_{\mathrm{GRB}}(0)$ is the overall GRB rate at $z=0$ where the geometrically correction is taken into account. $f_{\mathrm{F}} \xi_{\text {acc }}$ expresses the ratio of the nonthermal baryon energy of flares to the prompt radiated energy. If the nonthermal baryon energy is comparable to $E_{\mathrm{GRB}}$, we can expect a significant contribution to the neutrino background from flares. Our numerical results obtained by the same method as in our previous paper are shown in Fig. 3 for the cases of $f_{\mathrm{F}} \xi_{\text {acc }}=(0.5-5)$. Expected muon events from above $\mathrm{TeV}$ neutrinos are also shown in Fig. 3. Although the expected muon events of above $\mathrm{TeV}$ neutrinos from flares will be smaller than those from prompt bursts (shown in Fig. 3), they can be comparable with or exceed those from prompt bursts in the very high energy region above a few $\mathrm{PeV}$ range.

Implications and discussions. - In the near future, high energy neutrino signals from flares may be detected by IceCube and/or Auger which can provide information on the nature of flares. We expect such neutrino flashes from flares should be in coincidence with the early afterglow phase. Especially, some of events will be correlated with observed flares. These signals may be expected from not only long GRBs but also short GRBs, that also may accompany flares [6], if such flares are baryonic origins. However, several authors recently discussed flares may not be of baryonic origins but of magnetic origins [16]. If the outflow is much magnetized, formed shocks would be greatly weakened and neutrino emission would be suppressed [16]. The detection of high energy neutrinos is one of the tests for the origin of flares. In addition, these signals would give us information not only on the magnetic field but also on the photon field. As demonstrated in FUVray flare (B) in Fig. 2, too copious photon fields will reduce the maximum proton energy and the following maximum neutrino energy. Moreover, such neutrino detection may include signals from FUV-ray flares that are not seen due to absorption by neutral hydrogen both in host galaxy and in our Galaxy. Combined with gamma-ray large area space telescope (GLAST) mission which may detect sub-GeV flashes by IC [7] or $\pi^{0}$ decay, these high energy neutrinos may be important as a probe of FUV-ray emissions.

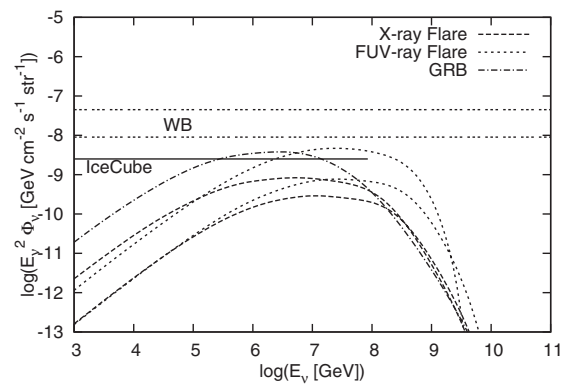

FIG. 3. The neutrino background from flares. X-ray flare (the upper dashed line): $L_{\max }=10^{49} \mathrm{ergs} / \mathrm{s}, \xi_{B}=1, f_{\mathrm{F}} \xi_{\mathrm{acc}}=1$, and $r=10^{15.3} \mathrm{~cm}$ with $\Gamma=15 ; N_{\mu}=3.7$ events/yr. (The lower dashed line): $L_{\max }=10^{49} \mathrm{ergs} / \mathrm{s}, \xi_{B}=1, f_{\mathrm{F}} \xi_{\mathrm{acc}}=0.5$, and $r=$ $10^{14.9} \mathrm{~cm}$ with $\Gamma=30 ; N_{\mu}=0.8$ events $/ y r$. FUV-ray flare (the upper dotted line): $L_{\max }=10^{49} \mathrm{ergs} / \mathrm{s}, \xi_{B}=0.1, f_{\mathrm{F}} \xi_{\text {acc }}=5$, and $r=10^{14.9} \mathrm{~cm}$ with $\Gamma=30 ; N_{\mu}=8.8$ events $/ \mathrm{yr}$. (The lower dotted line): $L_{\max }=10^{48} \mathrm{ergs} / \mathrm{s}, \xi_{B}=1, f_{\mathrm{F}} \xi_{\text {acc }}=1$, and $r=$ $10^{15.3} \mathrm{~cm}$ with $\Gamma=15 ; N_{\mu}=1.4$ events $/ y r$. GRB: taken from [2] with $E_{\gamma, \mathrm{sh}}^{\text {iso }}=2 \times 10^{51} \mathrm{ergs}, \xi_{B}=1, \quad \xi_{\text {acc }}=10$, and $r=$ $\left(10^{13}-10^{14.5}\right) \mathrm{cm} ; N_{\mu}=21$ events/yr. WB: Waxman-Bahcall bounds [1]. 
We have estimated the flux of neutrinos from flares by normalizing the proton flux with the typical prompt radiated energy. For the capability of detections, outflows may need to be largely baryon loaded. Many flares are likely to be interpreted as the late activity of the central engine. Such an energy injection is also one of the common interpretations for early flattening of the x-ray afterglow and its energy will be comparable to that of the prompt burst [5]. These late activities might supply the extra nonthermal baryons. However, too large nonthermal baryon loading will not be plausible [2] and would also be constrained by high energy $\gamma$ rays. The high energy $\gamma$ rays could cascade in the source and/or in infrared and microwave background where the delayed emission would occur [17]. The delayed emission, if it occurs, would be expected in the $\mathrm{GeV}-\mathrm{TeV}$ region and extended to the keV-MeV region. In addition, there would also be contributions of synchrotron radiation components from charged particles such as pions. Although the detailed calculation is needed to obtain reliable spectra, such emission could not be detected unless the source is very nearby and/or the flare is energetic. [For example, in the case of the set with $L_{\max }=10^{49} \mathrm{ergs} / \mathrm{s}, r=10^{14.9} \mathrm{~cm}$, and $\Gamma=30$, the inferred spectra [17] imply that the detection by burst alert telescope would be difficult at $z \gtrsim 0.1$.] GLAST would be able to test the existence of high energy emission.

At present, we do not know the total radiated energy from flares very well. The fluences of x-ray flares are usually smaller than that of the bursts, but can be even comparable to or higher than that of the bursts. There are also some GRBs with multiple flares, and there might be a significant fraction of the FUV-ray flares. We also do not know the opening angle of flaring outflows themselves. If the late slow outflow might have the larger opening angle than the prompt fast outflow, of which beaming factor $f_{b}=$ $E_{\mathrm{GRB}} / E_{\gamma, \text { tot }}^{\text {iso }}$ is typically $\sim(0.01-0.1)$, we can expect high energy neutrinos uncorrelated with GRBs. Many parameters are still uncertain but these distributions are important for more realistic predictions. For larger collision radii with $r \sim 10^{16} \mathrm{~cm}$, we expect fewer neutrinos. Therefore, our evaluation could be maybe applied to about only a half of flares, and the other half would make $\mathrm{GeV}$ flares and very high energy cosmic rays. Unknown features of flares will be unveiled through more multiwavelength observations by Swift, GLAST and so on. We expect future neutrino observations will also give us some clues on the physical parameters of flares.

Waxman and Bahcall [1] predicted neutrino burst under the assumption that GRBs are the main sources of ultrahigh-energy cosmic rays (UHECRs). In the case of the prompt bursts, the optical thickness for the photomeson production can be smaller than the unity especially at larger radii $r \gtrsim 10^{14}\left(E_{\gamma, \mathrm{sh}}^{\text {iso }} / 10^{51} \mathrm{ergs}\right)^{1 / 2} \mathrm{~cm}$ and the UHECRs can be produced in such regions. In flares, it is more difficult to generate UHECRs especially in the case of FUV-ray flares where the optical thickness for the photo- meson production can be larger. So far, we have not taken account of neutrino oscillations. Actually, neutrinos will be almost equally distributed among flavors as a result of vacuum neutrino oscillations [1].

We thank K. Ioka, K. Toma, K. Asano, and Z. G. Dai for many profitable suggestions. S. N. is partially supported by Grants-in-Aid for Scientific Research from the Ministry of E.C.S.S.T. of Japan through No. 16740134.

[1] E. Waxman and J. Bahcall, Phys. Rev. Lett. 78, 2292 (1997); Phys. Rev. D 59, 023002 (1998).

[2] K. Murase and S. Nagataki, Phys. Rev. D 73, 063002 (2006).

[3] E. Waxman and J. Bahcall, Astrophys. J. 541, 707 (2000); Z. G. Dai and T. Lu, Astrophys. J. 551, 249 (2001); C. D. Dermer, Astrophys. J. 574, 65 (2002); C. D. Dermer and A. Atoyan, Phys. Rev. Lett. 91, 071102 (2003); D. Guetta, D. W. Hooper, J. Alvarez-Muñiz, F. Halzen, and E. Reuveni, Astropart. Phys. 20, 429 (2004); K. Asano, Astrophys. J. 623, 967 (2005).

[4] B. Zhang and P. Mészáros, Int. J. Mod. Phys. A 19, 2385 (2004); T. Piran, Rev. Mod. Phys. 76, 1143 (2005).

[5] S. Kobayashi et al., astro-ph/0506157; B. Zhang et al., Astrophys. J. 642, 354 (2006); P. T. O'Brien et al., astro$\mathrm{ph} / 0601125$.

[6] D. N. Burrows et al., Science 309, 1833 (2005); S. Barthelmy et al., Nature (London) 438, 994 (2005); G. Cusumano et al., Nature (London) 440, 164 (2006); A.D. Falcone et al., Astrophys. J. 641, 1010 (2006); P. Romano et al., A \& A 450, 59 (2006).

[7] Y.Z. Fan and D. M. Wei, Mon. Not. R. Astron. Soc. 364, L42 (2006); Y.Z. Fan and T. Piran, Mon. Not. R. Astron. Soc. 370, L24 (2006).

[8] S. Agostinelli et al., Nucl. Instrum. Methods Phys. Res., Sect. A 506, 250 (2003).

[9] S. Schadmand, Eur. Phys. J. A 18, 405 (2003); Particle Data Group, http://pdg.lbl.gov/.

[10] ANTARES Collaboration, astro-ph/9907432. P. K. F. Grieder et al., Nuovo Cimento 24C, 771 (2001); J. Ahrens et al., Astropart. Phys. 20, 507 (2004).

[11] K. Ioka, S. Kobayashi, and B. Zhang, Astrophys. J. 631, 429 (2005).

[12] D. Proga and M. Begelman, Astrophys. J. 592, 767 (2003); A. King et al., Astrophys. J. 630, L113 (2005); R. Perna, P. J. Armitage, and B. Zhang, Astrophys. J. 636, L29 (2006).

[13] K. Asano and F. Takahara, Publ. Astron. Soc. Jpn. 55, 433 (2003); Z. Li and L. M. Song, Astrophys. J. 608, L17 (2004).

[14] F. C. Jones, Phys. Rev. 137, B1306 (1965).

[15] C. Porciani and P. Madau, Astrophys. J. 548, 522 (2001); D. Guetta, T. Piran, and E. Waxman, Astrophys. J. 619, 412 (2005).

[16] B. Zhang and S. Kobayashi, Astrophys. J. 628, 315 (2005); Y.Z. Fan et al., Astrophys. J. 635, L129 (2005); Z. G. Dai et al., Science 311, 1127 (2006); D. Proga and B. Zhang, Mon. Not. R. Astron. Soc. 370, L61 (2006).

[17] C. D. Dermer and A. Atoyan, A \& A 418, L5 (2004); S. Razzaque, P. Mészáros, and B. Zhang, Astrophys. J. 613, 1072 (2004). 